\title{
Editorial
}

\section{Scientific Advocacy: Confronting the Oxymoron}

\section{Activisme scientifique: un oxymoron auquel on doit faire face}

\author{
Marc-André Villard $^{1}$ and Thomas D. Nudds ${ }^{2}$
}

Scientists are often caught between their desire to take a stand in ecological debates and an equally strong urge to better understand the problem at hand. Noss $(1989,1994)$ argues that scientists have a professional responsibility to provide advice on policy issues, and that failing to advocate changes to current environmental policy when such changes are required based on science is akin to advocating status quo. Others argue that scientists who are involved in advocacy undermine their credibility and that of their discipline, because it polarizes debates and increases confrontation instead of achieving the partnerships required to address conservation issues (e.g., Brussard et al. 1994, Baskerville 1997). Advocacy may also affect the way scientists frame research questions or design studies. This, in turn, may yield weak scientific evidence, which is worthless in environmental debates (Wiens 1997).

Avian conservation ecologists are especially likely to be faced with the dilemma of having to provide advice to managers or policy makers. We are trained to ask the right questions, collect good quality data, and analyze them appropriately. A corollary to this training is that we want to avoid making hasty assessments based on incomplete information because this could actually make matters worse. The dilemma, of course, is that, on the one hand, to delay policy decisions until uncertainty is reduced and the system sufficiently understood may have dire consequences. On the other hand, like nature itself, our knowledge about uncertainty is also constantly in flux, and it persists regardless (see Nudds and Villard 2005). If the essence of sound conservation science is to embrace uncertainty, then how is it possible to advocate? Thus, many scientists are reluctant to join the ranks of environmental activists, or to accept research funds in partnership with industry, for fear that their credibility as objective analysts may suffer.

Scientists who do take up causes may risk rebuke or worse. For example, NASA scientists were recently reminded that they were free to discuss scientific findings, but policy statements should be left to policy makers and appointed spokespersons (Revkin 2006). The question is: where does science end and policy begin? The answer is that, in the radical middle ground of conservation science, there is no dichotomy between science and policy and, thus, no end to one and beginning to the other. Rather, as Karl Popper recognized (Magee 1985:75), "Policy is a hypothesis that needs to be revised in the light of experience." Or, to paraphrase Carpenter et al. (1999:18), "Frozen policy is a route to disaster." We contend that the topic of advocacy science remains hotly debated, and positions rather entrenched, precisely because scientists have not generally embraced this philosophical stance; at Avian Conservation and Ecology, we do.

This view of the policy-science relationship is, in essence, equivalent to adaptive management (e.g., Lancia et al. 1996, Nudds 1998, and many others). In practice, however, cases of adaptive management/policy making remain extremely rare and in most jurisdictions, policy making does not reflect current ecological understanding (Wallington et al. 2005). Therefore, it is critical that scientists

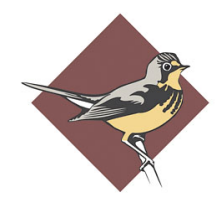

BIRD STUDIESCANADA
ETUDES D'OISEAUXCANA 
communicate their findings in a clear and efficient manner directly to policy makers, to the public (Wiens 1997, Wallington et al. 2005), and to each other. Papers published in Avian Conservation and Ecology should address this challenge by outlining in detail how ecological insight relates to current policy, or how current policy influences ecosystems in which birds are a focal taxon. We encourage contributors at least to reflect upon this link a posteriori, if not at the project development stage. Our open-access, online format is ideal to influence the type of research and policy making that we are promoting above. By allowing for rapid, efficient, and free dissemination of knowledge, we wish to contribute to the adaptive learning process, which depends on reliable scientific knowledge. Researchers have a responsibility to communicate their results efficiently, in a fashion that is both understandable and useful to practitioners. In a nutshell, to paraphrase Wiens (1997:2) "...our agenda [as ecologists] should be science, and our responsibility is to ensure that [reliable] scientific findings carry the greatest possible weight in societal decisions about the environment."

Les chercheurs scientifiques sont souvent pris entre leur désir de prendre position dans les débats écologiques et un besoin aussi pressant de mieux comprendre les problématiques en question. Noss (1989, 1994) soutient que, pour les scientifiques, fournir un avis sur les politiques environnementales constitue une responsabilité professionnelle et que le fait de ne pas exiger des changements à ces politiques lorsque la science indique qu'ils sont nécessaires revient à promouvoir le statu quo. D'autres soutiennent que les scientifiques impliqués dans l'activisme entachent leur crédibilité et celle de leur discipline, parce que ceci polarise les débats et augmente la confrontation plutôt que de générer les partenariats requis afin de s'attaquer aux problèmes de conservation (ex., Brussard et al. 1994, Baskerville 1997). L'activisme peut également influencer la façon dont les chercheurs formulent leurs questions de recherche ou conçoivent leurs projets de recherche. Ceci résulte en une faible capacité d'inférence, ce qui ne contribue aucunement à enrichir les débats environnementaux (Wiens 1997).

Les spécialistes de l'écologie et de la conservation des oiseaux sont particulièrement susceptibles d'être confrontés au dilemme ci-haut mentionné lorsqu'ils sont consultés par les aménagistes ou les responsables de l'élaboration des politiques. Nous sommes formés pour poser les bonnes questions, récolter des données de qualité et les analyser de façon appropriée. En corollaire, nous évitons de poser des jugements hâtifs fondés sur des données incomplètes ou inadéquates parce que ceci pourrait empirer la situation. Bien sûr, ceci pose le dilemme suivant : d'une part, repousser les décisions jusqu'à ce que l'incertitude soit réduite et le système mieux compris risque d'entraîner des conséquences fâcheuses. D'autre part, comme la nature elle-même, nos connaissances sur celle-ci sont en constante évolution et l'incertitude est une caractéristique inhérente à l'étude des écosystèmes (Nudds et Villard 2005). Si l'essence même de la science de la conservation est de faire face à l'incertitude, alors comment est-il possible de prendre position? De nombreux scientifiques sont donc peu enclins à joindre les rangs des activistes environnementaux, ou à accepter des fonds de recherche en partenariat avec l'industrie, de peur que leur crédibilité en tant qu'analystes objectifs en souffre.

Les scientifiques qui prennent fait et cause pour un dossier précis risquent d'essuyer une rebuffade ou pire encore. Par exemple, la NASA a récemment rappelé à ses chercheurs qu'ils et elles étaient libres de discuter de leurs découvertes mais que les énoncés politiques relevaient des fonctionnaires responsables des politiques et des porte-parole officiels (Revkin 2006). La question est donc la suivante : où la responsabilité scientifique se termine-t-elle et où les responsables de l'élaboration des politiques prennent-ils le relai? La réponse est que dans l'entre-deux radical que constitue la conservation, il n'y a pas de dichotomie entre la science et les politiques, donc pas de fin à l'une ni de début aux autres. Plutôt, tel que noté par Karl Popper (Magee 1985:75), « la politique est une hypothèse qui doit être révisée à la lumière de l'expérience ». Ou encore, pour citer Carpenter et al., (1999), « une politique figée dans le temps mène vers le désastre. » Nous soutenons que le thème de l'activisme scientifique demeure hautement controversé et que les positions de chaque camp demeurent tranchées, précisément parce que les chercheurs n'ont généralement pas adopté cette position philosophique. Écologie et conservation des oiseaux y adhère.

Cette vision de la relation entre la science et la politique est essentiellement équivalente à l'aménagement adaptatif (ex., Lancia et al. 1996, 
Nudds 1998 et plusieurs autres). En pratique, toutefois, l'aménagement et le développement « adaptatifs » de politiques demeurent extrêmement rares et, dans la plupart des juridictions, les politiques environnementales ne reflètent pas les connaissances écologiques actuelles (Wallington et al. 2005). Il est donc essentiel que les scientifiques communiquent leurs découvertes de façon claire et efficace, directement aux responsables de l'élaboration des politiques, au public (Wiens 1997 , Wallington et al. 2005) et aux autres chercheurs. Les articles publiés dans Écologie et conservation des oiseaux devraient faire face à ce défi en indiquant clairement en quoi les avancées écologiques qu'ils présentent sont pertinentes en regard des politiques actuelles ou de quelle façon les politiques actuelles influencent les écosystèmes dans lesquels les oiseaux représentent un taxon ciblé. Nous encourageons les auteurs à réfléchir à ce lien au moins a posteriori si ce n'est au moment du développement du projet. Notre politique d'accès libre en ligne est idéale pour influencer le type de recherche et d'élaboration de politiques dont nous faisons la promotion ci-haut. En permettant une diffusion rapide, efficace et gratuite des connaissances, nous souhaitons contribuer au processus adaptatif d'apprentissage qui dépend de connaissances scientifiques solides. Les chercheurs ont la responsabilité de communiquer leurs résultats efficacement, de façon compréhensible et utile pour les utilisateurs éventuels de ces connaissances. Bref, comme le dit Wiens (1997) « ...notre objectif [en tant qu'écologistes] devrait être de faire de la science et notre responsibilité est de nous assurer que nos résultats scientifiques les plus robustes influencent le plus possible les décisions que prend la société par rapport à l'environnement ».

Responses to this article can be read online at: http://www.ace-eco.org/voll/iss2/art5/responses/

\section{LITERATURE CITED}

Baskerville, G. L. 1997. Advocacy, science, policy, and life in the real world. Conservation Ecology 1 (1):9. [online] URL: http://www.consecol.org/vol1/ iss $1 /$ art $9 /$.

Brussard, P. F., D. D. Murphy, and C. R. Tracy. 1995. Grazing and advocacy. Conservation Biology 9:235-236.
Carpenter, S., W. Brock, and P. Hanson. 1999. Ecological and social dynamics in simple models of ecosystem management. Conservation Ecology 3(2):4. [online] URL: http://www.consecol.org/vol3/ iss $2 / \operatorname{art} 4 /$.

Lancia, R. A., C. E. Braun, M. W. Collopy, R. D. Dueser, J. G. Kie, C. J. Martinka, J. D. Nichols, T. D. Nudds, W. R. Porath, and N. G. Tilghman. 1996. ARM! For the future. Wildlife Society Bulletin 24:436-442.

Magee, B. 1985. Popper. Third edition. Fontana, London, UK.

Noss, R. F. 1989. Who will speak for biodiversity? Conservation Biology 3:202-203.

Noss, R. F. 1994. Cows and conservation. Conservation Biology 8:613-616.

Nudds, T. D. 1998. Adaptive management and the conservation of biodiversity. Pages 179-193 in R. K. Baydack, H. Campa III, and J. B. Haufler, editors. Practical approaches to the conservation of biodiversity. Island Press, Covelo, California, USA.

Nudds, T. D., and M.-A. Villard. 2005. Basic science, applied science, and the radical middle ground. Avian Conservation and EcologyÉcologie et conservation des oiseaux 1(1):1. [online] URL: http://www.ace-eco.org/vol1/iss1/art1/.

Revkin, A. C. 2006. 29 January. Climate expert says NASA tried to silence him. The New York Times 1:1. Available online at: http://www.nytimes.com/2006 /01/29/science/earth/29climate.html?ei $=5088 \&$ en $=$ 28e236da0977ee7f\&ex $=1296190800 \&$ pagewanted $=$ all/.

Wiens, J. A. 1997. Scientific responsibility and responsible ecology. Conservation Ecology 1 (1):16. [online] URL: http://www.consecol.org/vol1/ iss1/art16/.

Willington, T. J., R. J. Hobbs, and S. A. Moore. 2005. Implications of current ecological thinking for biodiversity conservation: a review of the salient issues. Ecology and Society 10(1):15. [online] URL: http://www.ecologyandsociety.org/vol10/iss 1/ art15/. 